\title{
Proteome Analysis of Date Palm (Phoenix dactylifera L.) under Severe Drought and Salt Stress
}

\author{
Haddad A. El Rabey, ${ }^{1,2}$ Abdulrahman L. Al-Malki, ${ }^{1}$ and Khalid O. Abulnaja ${ }^{1}$ \\ ${ }^{1}$ Biochemistry Department, Faculty of Science, King Abdulaziz University, Jeddah, Saudi Arabia \\ ${ }^{2}$ Bioinformatics Department, Genetic Engineering and Biotechnology Institute, Sadat City University, Sadat City, Minufya, Egypt \\ Correspondence should be addressed to Haddad A. El Rabey; elrabey@hotmail.com
}

Received 9 May 2016; Revised 11 August 2016; Accepted 8 September 2016

Academic Editor: Mohamed Salem

Copyright ( 2016 Haddad A. El Rabey et al. This is an open access article distributed under the Creative Commons Attribution License, which permits unrestricted use, distribution, and reproduction in any medium, provided the original work is properly cited.

Date palm cultivars differently tolerate salinity and drought stress. This study was carried out to study the response of date palm to severe salinity and drought based on leaf proteome analysis. Eighteen-month-old date palm plants were subjected to severe salt $(48 \mathrm{~g} / \mathrm{L} \mathrm{NaCl})$ and drought $(82.5 \mathrm{~g} / \mathrm{L} \mathrm{PEG}$ or no irrigation) conditions for one month. Using a protein 2D electrophoresis method, 55 protein spots were analyzed using mass spectrometry. ATP synthase CF1 alpha chains were significantly upregulated under all three stress conditions. Changes in the abundance of RubisCO activase and one of the RubisCO fragments were significant in the same spots only for salt stress and drought stress with no irrigation, and oxygen-evolving enhancer protein 2 was changed in different spots. Transketolase was significantly changed only in drought stress with PEG. The expression of salt and drought stress genes of the chosen protein spots was either overexpressed or downexpressed as revealed by the high or low protein abundance, respectively. In addition, all drought tolerance genes due to no irrigation were downregulated. In conclusion, the proteome analysis of date palm under salinity and drought conditions indicated that both salinity and drought tolerance genes were differentially expressed resulting in high or low protein abundance of the chosen protein spots as a result of exposure to drought and salinity stress condition.

\section{Introduction}

Plant species differ in their tolerance to abiotic stress. Salt sensitivity causes both rapid osmotic phase inhibiting growth of young leaves and a slower, ionic phase accelerating senescence of mature leaves causing reduction in crop plants yield [1]. Proteome analysis is a convenient tool for testing the response of plants to abiotic stress [2,3]. Date palm (Phoenix dactylifera L.) can adapt to extreme drought, heat, and relatively high levels of soil salinity [4]. Date palms can grow under a variety of environmental conditions such as heat, and water shortage and salinity of the ground water provide abiotic stresses which decrease date production [5].

Plants respond to a stress by modulating abundance of candidate proteins, either by upregulating expression or by the synthesizing novel proteins primarily associated with plant defense system [6]. Therefore, proteome of plant species changes as a response to biotic "pests" [7-9] and abiotic "chemical, salt, and drought stress" [3, 10, 11]. Proteome analysis of roots and leaves revealed a synergetic responsive network under stress; roots rapidly sensed and responded to stress, after which the stress signals were transferred to leaves and both roots and leaves showed similar metabolic pathways under stress with distinct changes [12]. Under salinity, salt is transported via the xylem to the shoot causing the accumulation of $\mathrm{Na}^{+}$and $\mathrm{Cl}^{-}$ions in shoot cells to a toxic extent, resulting in ionic stress that leads to ion accumulation enhancing the production of reactive oxygen species (ROS). The extra production of ROS in cells leads to a disruption of the balance between production and removal of ROS which eventually leads to oxidative stress [13]. Paul et al. [14] stated that the major class of identified proteins resulting from salinity stress response belongs to carbohydrate and energy metabolism category while stress and defense related proteins are especially up-accumulated under drought stress and a novel protein, "R40Cl", was reported to be up-accumulated 


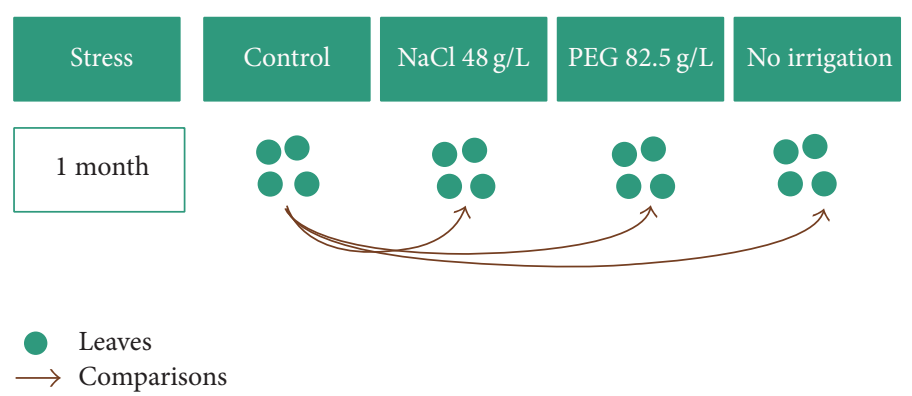

FIGURE 1: Scheme of the performed proteome analysis.

in roots of transgenic plants which may play an important role in generation of drought tolerant plants.

Recent proteome analyses have identified numerous drought-responsive proteins, which are involved in redox regulation, oxidative stress response, signal transduction, protein folding, secondary metabolism, and photosynthesis $[15,16]$. Production of proline is a common response to various abiotic stresses and its differential accumulation cannot be used as a molecular marker in date palm breeding programs aimed at improving drought or salinity tolerance traits in date palms. This conclusion is consistent with the theory that the molecular outcomes of abiotic stresses are often nonspecific [17].

This study aimed to analyze proteome of eighteen-monthold date palm plants subjected to severe salt $(48 \mathrm{~g} / \mathrm{L} \mathrm{NaCl})$ and drought (82.5 g/L PEG or no irrigation) conditions for one month. Changes in proteins levels under salinity and drought stress helped in defining genes correlated with these stress conditions that encoded these proteins.

\section{Materials and Methods}

2.1. Stress Experiment. Eighteen-month-old plants of date palm originated from tissue culture were subjected to three stress conditions for 30 days. The stress conditions were salt stress using $48 \mathrm{~g} / \mathrm{L} \mathrm{NaCl}$, drought using $82.5 \mathrm{~g} / \mathrm{L}$, and drought due to no irrigation. The experiment was terminated, when nonirrigated samples started to wilt and the other treatments showed dwarfism and reduction in the leaf area compared to the control plants. Salt stress samples $(76,77,78$, and 80 , see Table 1), 4 drought stress samples from PEG treatment (86, 88,89 , and 90), 4 drought stress samples with no irrigation $(61,62,63$, and 64), and 4 control samples at the end of the experiment (51,52, 54, and 55) were chosen for proteome analysis as shown in Figure 1.

2.2. Protein Extraction, Labeling, and 2D Electrophoresis. Protein was extracted according to the method described in El Rabey et al. [11]. Briefly, four replicates of the frozen shoot of stressed and control plants were ground into a fine powder in liquid nitrogen. Proteins were precipitated by the addition of $1.8 \mathrm{~mL}$ of ice-cold acetone containing $0.07 \%$ $(\mathrm{v} / \mathrm{v})$ mercaptoethanol. One hundred $\mathrm{mg}$ of each sample was dissolved in $400-600 \mu \mathrm{L}$ of IEF buffer (7 M urea, $2 \mathrm{M}$ thiourea, 2\% (w/v) CHAPS, and $30 \mathrm{mM}$ Tris, $\mathrm{pH} 8.0)$. The
TABLE 1: Labeling scheme for 8 gels: the internal standard (IS) and each analyzed sample were labeled with CyDye 2, 3, or 5 as shown in the table. The internal standard is generally the mixture of the same portions of all analyzed samples.

\begin{tabular}{lccc}
\hline Gel number & CyDye 2 & CyDye 3 & CyDye 5 \\
\hline 1 & IS & $88^{\prime}$ & $51^{*}$ \\
2 & IS & $61^{\wedge}$ & $52^{*}$ \\
3 & IS & $54^{*}$ & $76^{\#}$ \\
4 & IS & $55^{*}$ & $86^{\prime}$ \\
5 & IS & $77^{\#}$ & $62^{\wedge}$ \\
6 & IS & $89^{\prime}$ & $64^{\wedge}$ \\
7 & IS & $63^{\wedge}$ & $90^{\prime}$ \\
8 & IS & $80^{\#}$ & $78^{\#}$ \\
\hline
\end{tabular}

*: control, \#: PEG drought stress, $\wedge$ : no irrigation drought stress, and ": salt stress.

proteins were resolubilized overnight at room temperature. The mixture was then centrifuged for 10 minutes at $4^{\circ} \mathrm{C}$ at $16,100 \times \mathrm{g}$ and total soluble protein in the supernatants was quantified using the 2D Quant Kit (GE Healthcare, Munich, Germany). Labeling and mixing scheme for the performed DIGE experiment is shown in Table 1. Fifty micrograms of total soluble protein was used for analysis of each sample in 2D gel. Eight analytical gels were run in $\mathrm{pH}$ range 4-7. Part of the internal standard was saved before labeling with CyDye 2 and this unlabeled part was applied to 2 preparative gels that were in the end used for protein identification by mass spectrometry. Proteins from the extracts were separated in the first dimension according to their pI using IEF on Immobiline DryStrip, $24 \mathrm{~cm}, \mathrm{pH} 4-7$, and in the second dimension according to their molecular weight using SDSPAGE. All the 8 analytical gels and the 2 preparative gels were run at the same time.

After protein separation, the gels were scanned. Three fluorescence scans for each analytical gel were acquired and used for the analysis. The preparative gels were fixed for 1 hour in fixation solution (40\% ethanol and $10 \%$ acetic acid in water) and then stained with RuBPS ( $1 \mathrm{mM}$ in fixation solution) for 20 minutes and destained overnight in fixation solution. The preparative gels were scanned directly after destaining and stored wet at $4^{\circ} \mathrm{C}$ before spot cutting.

2.3. Biological Variation Analysis (BVA). BVA allowed quantitative comparisons of protein expression across multiple 

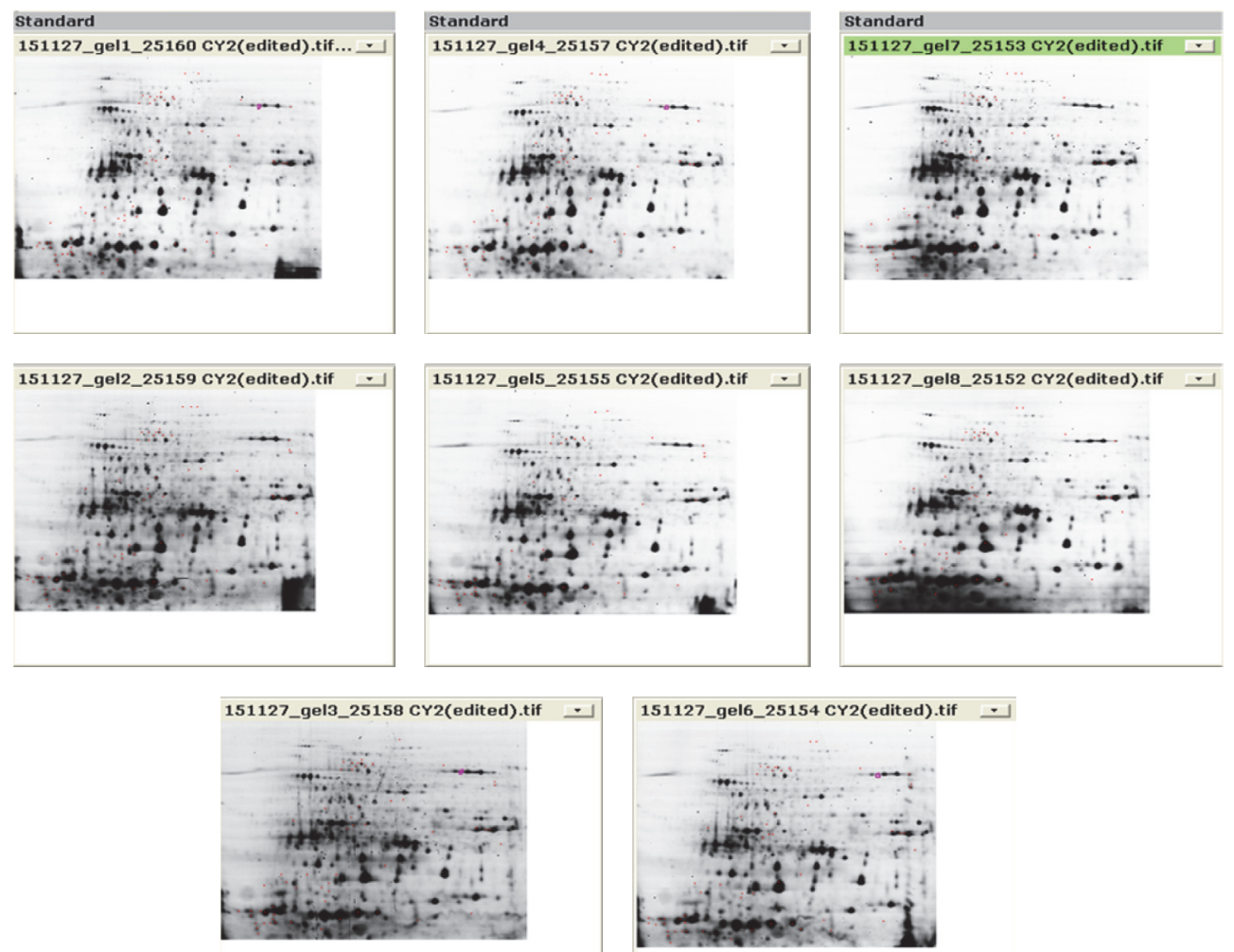

FIGURE 2: Overview of the fluorescence scans, internal standards.

gels. $t$-test ( $p$ value calculated using Student's $t$-test) and average ratio (fold difference in protein abundance of one protein spot between the groups selected in the protein statistics) values were calculated for all matched spots. The spots were filtered for $t$-test $p$ value lower than 0.05 and Av. ratio $>2$ and $<-2$. All automatically chosen spots were checked manually if they are real spots and marked for picking in the scan of the preparative gel.

\subsection{Identification of Proteins in the Spots of Interest Chosen} by DIGE Analysis. 55 spots were cut from the preparative gel for MS analysis. Proteins in the gel plugs were reduced with $10 \mathrm{mM}$ dithiothreitol and alkylated using $55 \mathrm{mM}$ iodoacetamide in $0.1 \mathrm{M}$ to open S-S bridges for action of trypsin. Digestion with trypsin $(12.5 \mathrm{ng} / \mu \mathrm{L}$ of trypsin in $50 \mathrm{mM}$ $\mathrm{NH}_{4} \mathrm{HCO}_{3}$ ) was performed overnight at $37^{\circ} \mathrm{C}$. The resulting peptides were extracted from the gel plugs in two extraction steps: first one with $25 \mathrm{mM} \mathrm{NH}_{4} \mathrm{HCO}_{3}$ and second one with $5 \%$ formic acid. Collected extracts were dried down and resolubilized in $2 \%$ acetonitrile with $0.1 \%$ formic acid in water (MS grade) for MS analysis. The resulting peptides were separated according to their hydrophobicity by nanoHPLC (C18 column, UltiMate 3000 HPLC system, Dionex) and sprayed directly into an ion trap spectrometer (amaZon ETD, Bruker Daltonics) using nanoESI sprayer.

2.5. Data Mining. Processed MS/MS spectra were used for the protein identification with in-house Mascot Search server (Matrix Science software, Matrix Science Ltd., London, UK).
Swiss Prot (all species) and NCBInr (Green plants) databases were involved in the protein search.

\section{Results}

3.1. Results of 2D-Gel Electrophoresis. Figures 2, 3, and 4 show the three fluorescence scans for each analytical gel used for the analysis, whereas Table 2 shows the results of filtered spots by $t$-test $p$ value lower than 0.05 and Av. ratio $>2$ and $<-2$. Supplementary Figure 5, in Supplementary Material available online at http://dx.doi.org/10.1155/2016/7840759, shows location of the automatically chosen spots that were checked manually if they are real spots and marked for picking in the scan of the preparative gel.

3.2. DIGE Analysis. Table 2 illustrates detailed results of the 55 protein spots that were significantly changed as a result of salinity and drought stress as revealed by DIGE analysis. The results can be illustrated as follows: (i) in the salt stress plants, 15 spots showed higher protein abundance and 20 spots showed lower protein abundance in the leaves of the stressed plants compared to control plants of the same age; (ii) the drought stress with PEG produced three spots with higher protein abundance and 6 spots with lower protein abundance in the leaves of the stressed plants compared to control plants of the same age; (iii) drought stress with no irrigation did not produce any spots with higher protein abundance in spite of producing 19 spots with lower protein abundance in the leaves of the stressed plants compared to control plants of 

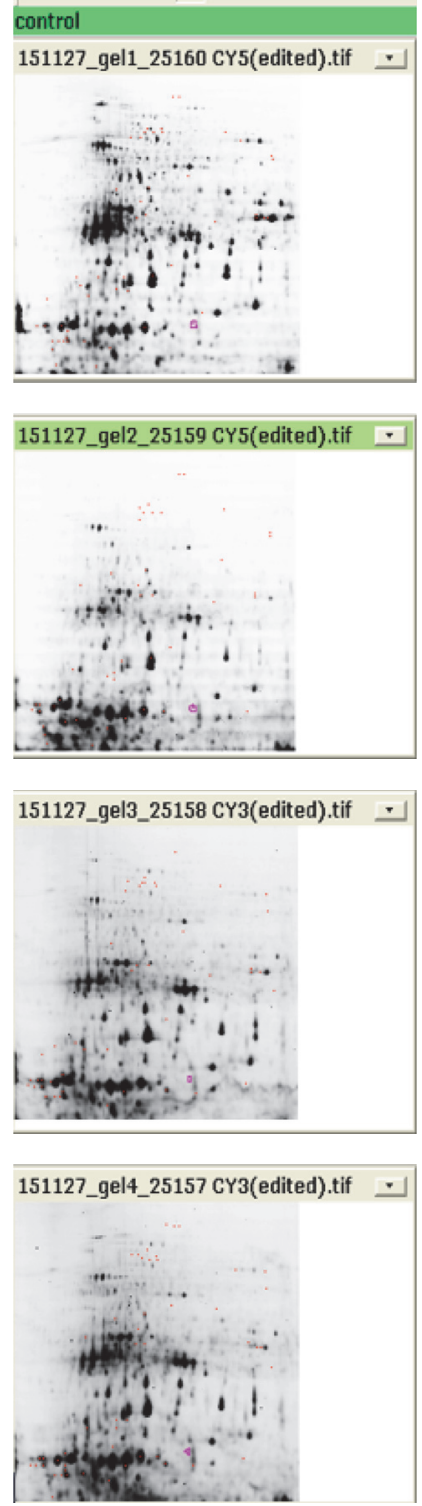
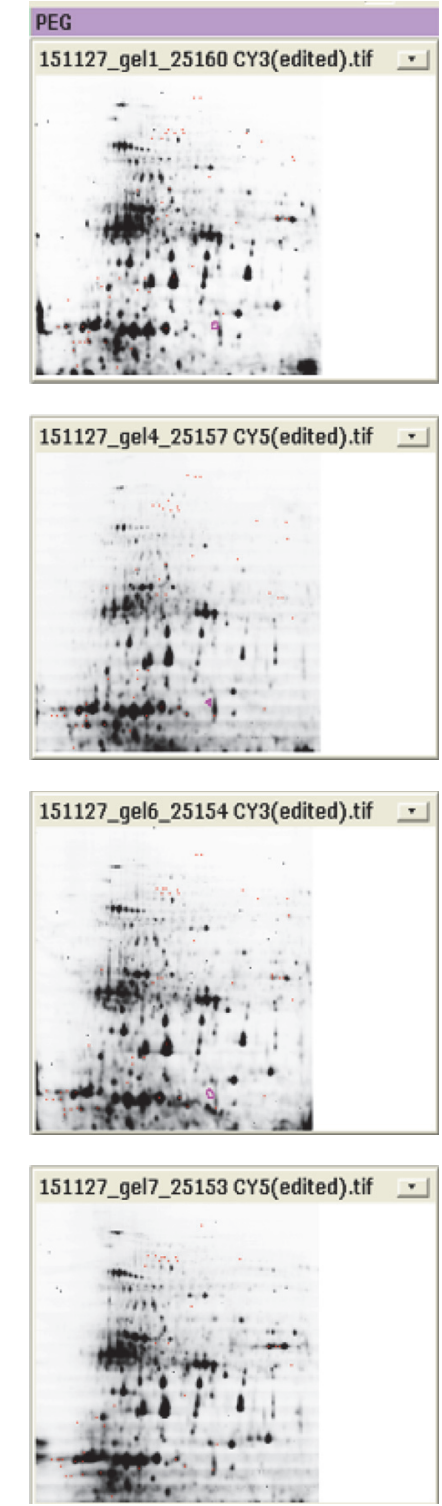
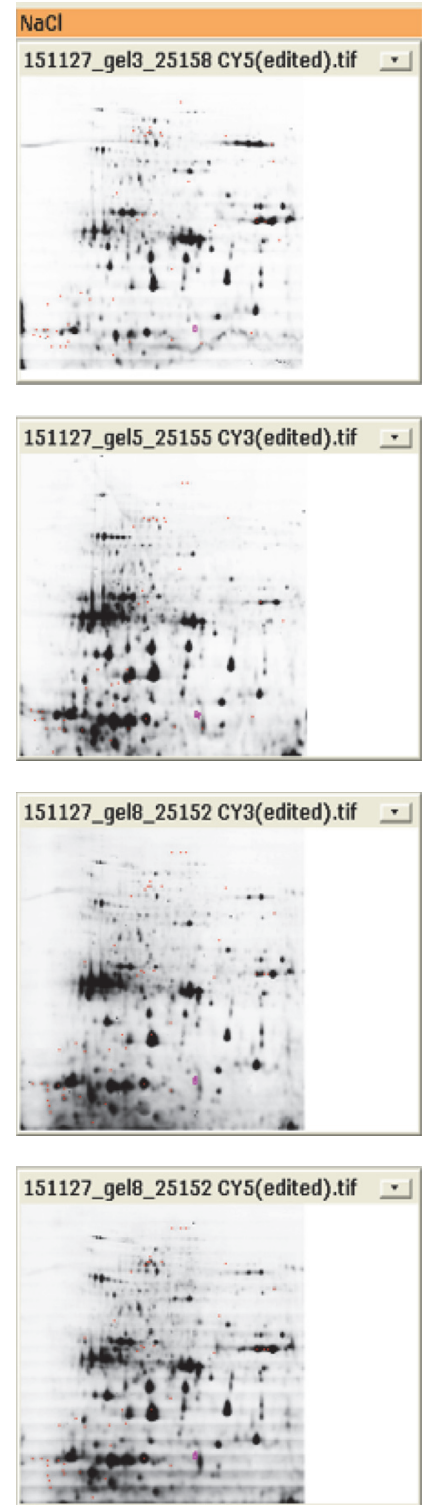
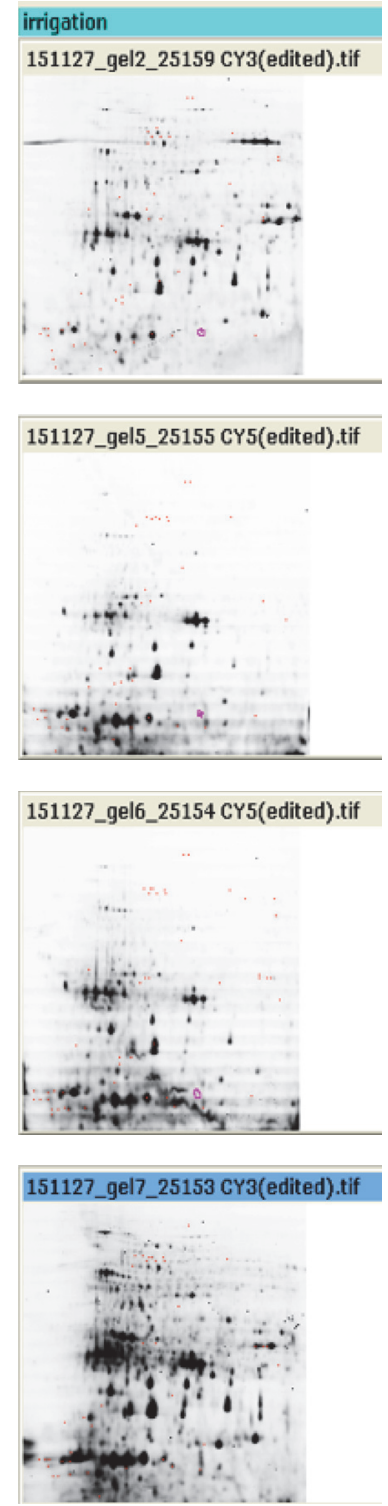

Figure 3: Overview of the fluorescence scans, analytical gels. Color code for analyzed samples: green, control; violet, PEG drought stress; orange, salt stress; blue, no irrigation drought stress.

the same age; (iv) six spots showed lower protein abundance in two studied stresses, salt stress and drought stress due to no irrigation; (v) only 1 spot showed lower protein abundance in all three studied stresses.

The 55 spots of Table 2 were chosen for protein identification by mass spectrometry, bigger portion of them with lower protein abundance under stress conditions compared to the unstressed control as revealed above.

3.3. MS Analysis and Data Mining. The results of MS analysis of the chosen 55 spots showed that 42 spots out of them were analyzed, whereas the other 13 were not analyzed due to no protein hit or not analyzed by MS (see the Supplemented Excel File). A BLAST-p search of the resulting protein sequence was done using NCBInr database/Green plants database for homologous protein search.
The spots with higher protein abundance in PEG treated group (which are listed in Table 2) were ribulose-1,5bisphosphate carboxylase/oxygenase (RubisCO) large subunit [Zantedeschia aethiopica] and transketolase (TRK), chloroplastic [Phoenix dactylifera], whereas those with high protein abundance in the $\mathrm{NaCl}$ treated group were uncharacterized protein LOC103705614 [Phoenix dactylifera], chaperonin hsp60 [Arabidopsis thaliana], chaperonin CPN60-2, mitochondrial-like [Phoenix dactylifera], serine acetyltransferase 1, chloroplastic-like [Brassica napus], betaglucosidase 12-like [Phoenix dactylifera], ATP synthase CF1 alpha chain (ATPase) [Phoenix dactylifera], ATP synthase subunit beta, mitochondrial-like [Phoenix dactylifera], ATP synthase beta subunit [Toronia toru], serine acetyltransferase 1 (SAT1), chloroplastic-like [Brassica napus], and ribulose-1,5bisphosphate carboxylase. 

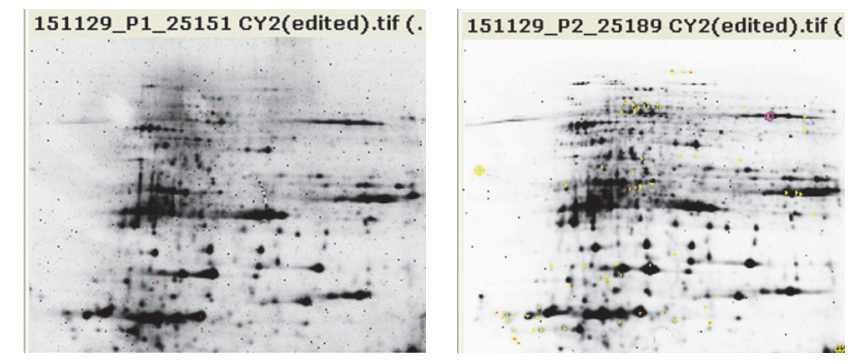

FIGURE 4: Overview of the fluorescence scans, preparative gels.

The distribution of the MS analyzed 42 spots that were significantly changed $(p<0.05)$ as a result of salinity and drought stress using either PEG or no irrigation compared to the control is as follows:

(i) Four proteins were significantly changed in all studied salinity and drought treatments (oxygenevolving enhancer protein 1 (OEE1), chloroplastic [Phoenix dactylifera], elongation factor TuA (EFTuA), chloroplastic-like [Phoenix dactylifera], ATP synthase CF1 alpha chain [Phoenix dactylifera], and ribulose1,5-bisphosphate carboxylase/oxygenase large subunit [Villarsia albiflora]).

(ii) Nine proteins were changed in both no irrigation and $\mathrm{NaCl}$ treated groups (ribulose-1,5-bisphosphate carboxylase/oxygenase large subunit, partial (chloroplast) [Ulva lactuca], ribulose bisphosphate carboxylase/oxygenase activase 2, chloroplastic isoform X1 [Phoenix dactylifera], ribulose bisphosphate carboxylase/oxygenase activase 2, chloroplastic isoform X1 [Phoenix dactylifera], ribulose-1,5-bisphosphate carboxylase/oxygenase large subunit, partial (plastid) [Torilis japonica], ribulose-1,5-bisphosphate carboxylase/oxygenase, partial (chloroplast) [Eriophorum viridicarinatum], ribulose-1,5-bisphosphate carboxylase/oxygenase large subunit, partial (chloroplast) [Eucalyptus stoatei], ribulose-1,5-bisphosphate carboxylase/oxygenase large subunit [Pimelea longiflora subsp. eyrei], ribulose-1,5-bisphosphate carboxylase/ oxygenase large subunit (RubisCO) [Andreaea rothii], and ribulose-1,5-bisphosphate carboxylase [Euonymus bungeanus]).

(iii) ATP synthase subunit $d$, mitochondrial [Phoenix dactylifera] was found to be significantly changed only in drought stress with PEG and $\mathrm{NaCl}$ treated groups, whereas glucose-methanol-choline (GMC) oxidoreductase, putative [Ricinus communis] was found to be significantly changed only in drought stress either PEG or No Irrigation.

(iv) 17 other proteins were significantly changed only in $\mathrm{NaCl}$ treated group (ribulose-1,5-bisphosphate carboxylase [Moraea fugax], chlorophyll a-b binding protein 6, chloroplastic [Vitis vinifera], phosphoglycerate kinase (PGK), cytosolic [Glycine max], ribulose-1,5-bisphosphate carboxylase/oxygenase large subunit [Zantedeschia aethiopica], ribulose1,5-bisphosphate carboxylase, glyceraldehyde-3phosphate dehydrogenase B (GPDB), chloroplastic [Phoenix dactylifera], dehydroascorbate (DHA) reductase-like [Phoenix dactylifera], serine acetyltransferase 1 (SAT1), chloroplastic-like [Brassica napus], ATP synthase beta subunit [Toronia toru], ATP synthase subunit beta, mitochondrial-like [Phoenix dactylifera], ATP synthase CF1 alpha chain [Phoenix dactylifera], beta-glucosidase 12like [Phoenix dactylifera], chaperonin CPN60-2, mitochondrial-like [Phoenix dactylifera], chaperonin hsp60 [Arabidopsis thaliana], and uncharacterized protein LOC103705614 [Phoenix dactylifera]).

(v) Five proteins were significantly changed only under drought condition exerted by PEG (serine acetyltransferase 1, chloroplastic-like [Brassica napus], transketolase, chloroplastic [Phoenix dactylifera], ribulose-1,5-bisphosphate carboxylase/oxygenase large subunit [Zantedeschia aethiopica], oxygenevolving enhancer protein 2, chloroplastic-like [Phoenix dactylifera], and cytochrome P450, putative [Ricinus communis]).

(vi) Five more proteins were also significantly changed only under drought condition exerted by no irrigation (ribulose-1,5-bisphosphate carboxylase [Euonymus bungeanus], ribulose-1,5-bisphosphate carboxylase/ oxygenase large subunit [Zantedeschia aethiopica], ribulose-bisphosphate carboxylase [Lysichiton americanus], ribulose-1,5-bisphosphate carboxylase/oxygenase large subunit [Lunularia cruciata], and oxygen-evolving enhancer protein 2, chloroplasticlike [Phoenix dactylifera]).

\section{Discussion}

This study described the response of 18-month-old date palm plants to severe salinity $(48 \mathrm{~g} / \mathrm{L} \mathrm{NaCl})$ and drought stress exerted by PEG $(82.5 \mathrm{~g} / \mathrm{L})$ or no irrigation for one month as revealed by proteome analysis of leaves. Proteome analysis of roots and leaves revealed a synergetic responsive network under stress [12]. The DIGE analysis results showed that 55 protein spots were significantly changed as a result 
TABLE 2: DIGE results: overview of the significantly changed spots and their behavior in other studied stresses.

\begin{tabular}{|c|c|c|c|c|c|c|c|}
\hline \multirow{2}{*}{ Spot number } & \multicolumn{2}{|c|}{$\mathrm{NaCl} /$ control } & \multicolumn{2}{|c|}{ PEG/control } & \multicolumn{2}{|c|}{ Irrigation/control } & \multirow{2}{*}{ Enzyme abbreviation } \\
\hline & $t$-test & Av. ratio & $t$-test & Av. ratio & $t$-test & Av. ratio & \\
\hline 65 & $0.0082^{*}$ & $2.17^{\wedge}$ & 0.37 & 1.2 & 0.084 & 1.9 & No protein hit \\
\hline 67 & $0.02^{*}$ & $2.24^{\wedge}$ & 0.79 & 1.03 & 0.13 & 1.65 & No protein hit \\
\hline 69 & $0.025^{*}$ & $2.44^{\wedge}$ & 0.82 & -1.11 & 0.31 & 1.58 & LOC103705614 \\
\hline 299 & $0.025^{*}$ & $2.4^{\wedge}$ & 0.5 & -1.32 & & 2.6 & No protein hit \\
\hline 323 & $0.011^{*}$ & $2.41^{\wedge}$ & 0.44 & 1.2 & 0.15 & 1.74 & CPhsp60 \\
\hline 337 & $0.047^{*}$ & $2.16^{\wedge}$ & 0.65 & -1.35 & 0.54 & 1.62 & ATPase CF1 alpha \\
\hline 348 & $0.019^{*}$ & $3.32^{\wedge}$ & 0.64 & 1.06 & 0.37 & 1.65 & CPN60-2 \\
\hline 373 & $0.0011^{*}$ & $2^{\wedge}$ & 0.44 & -1.11 & 0.28 & 1.72 & No protein hit \\
\hline 383 & $0.0043^{*}$ & $2.42^{\wedge}$ & 0.46 & 1.36 & 0.31 & 1.36 & SATase1 \\
\hline 402 & $0.034^{*}$ & $2.47^{\wedge}$ & 0.83 & -1.16 & 0.73 & 1.11 & Beta-glucosidase \\
\hline 403 & $0.02^{*}$ & $2.71^{\wedge}$ & 0.77 & 1.14 & 0.63 & 1.18 & ATPase CF1 alpha \\
\hline 485 & $0.03^{*}$ & $2.14^{\wedge}$ & 0.69 & 1.12 & 0.087 & 2.1 & ATPase beta \\
\hline 497 & $0.0053^{*}$ & $2.25^{\wedge}$ & 0.9 & -1.02 & 0.059 & 2.09 & ATPase beta \\
\hline 529 & & 8.29 & $0.05^{*}$ & $-3.57^{*}$ & & -2.5 & RubisCO \\
\hline 731 & $0.019^{*}$ & $2.25^{\wedge}$ & 0.59 & -1.18 & 0.093 & 1.79 & SAT1 \\
\hline 967 & & -2.33 & $0.026^{*}$ & $-2.6^{*}$ & 0.26 & -1.7 & CYP450 \\
\hline 988 & $0.047^{*}$ & $-2.52^{*}$ & 0.061 & -1.72 & 0.36 & -1.31 & GPDB \\
\hline 1021 & 0.91 & -1 & $0.04^{*}$ & $-2.28^{*}$ & 0.9 & -1.04 & No protein hit \\
\hline $1119^{\#}$ & $0.011^{*}$ & $-2.1^{*}$ & 0.19 & -1.21 & $0.0031^{*}$ & $-2.72^{*}$ & RubisCO activase 2 \\
\hline $1126^{\#}$ & $0.037^{*}$ & $-2.65^{*}$ & 0.63 & -1.2 & $0.031^{*}$ & $-2.23^{*}$ & RubisCO activase 2 \\
\hline 1270 & 0.44 & -1.21 & 0.093 & -1.58 & $0.011^{*}$ & $-2.22^{*}$ & No protein hit \\
\hline 1338 & 0.06 & -1.39 & 0.25 & 1.15 & $0.0086^{*}$ & $-2.04^{*}$ & No protein hit \\
\hline 1417 & $0.015^{*}$ & $-2.1^{*}$ & 0.49 & -1.23 & 0.077 & -1.7 & No protein hit \\
\hline $1427 ”$ & $0.0033^{*}$ & $-2.61^{*}$ & $0.00043^{*}$ & -2.4 & $0.025^{*}$ & $-2.29^{*}$ & ATPase CF1 alpha \\
\hline 1433 & $0.026^{*}$ & $-2.28^{*}$ & 0.1 & -1.45 & 0.12 & -1.59 & No protein hit \\
\hline 1471 & 0.61 & -1.1 & 0.45 & -1.16 & $0.023^{*}$ & $-2.01^{*}$ & No protein hit \\
\hline 1485 & $0.038^{*}$ & $2.37^{\wedge}$ & 0.88 & 1.07 & 0.71 & 1.11 & RubisCO \\
\hline 1520 & 0.69 & -1.06 & 0.74 & 1.06 & $0.007^{*}$ & $-2.03^{*}$ & No MS result \\
\hline 2070 & 0.16 & -1.51 & 0.93 & 1.06 & $0.033^{*}$ & $-2.59^{*}$ & OEEP2 \\
\hline 2117 & $0.0055^{*}$ & $-2.12^{*}$ & 0.1 & -1.26 & 0.014 & -1.67 & RubisCO \\
\hline 2177 & 0.23 & -1.52 & 0.034 & -1.99 & $0.024^{*}$ & $-2.42^{*}$ & GMC oxidoreductase \\
\hline 2201 & 0.058 & -2.06 & $0.044^{*}$ & $-2.34^{*}$ & 0.062 & -1.53 & OEEP2 \\
\hline 2202 & $0.03^{*}$ & $-2.17^{*}$ & 0.2 & -1.23 & 0.022 & -1.7 & RubisCO \\
\hline 2222 & 0.57 & -1.08 & 0.9 & 1.02 & $0.0067^{*}$ & $-2.1^{*}$ & RubisCO \\
\hline 2267 & $0.0071^{*}$ & $-2.09^{*}$ & 0.009 & -1.82 & 0.2 & -1.43 & ATPase d \\
\hline 2440 & & 1.07 & $0.048^{*}$ & $2.31^{\wedge}$ & 0.67 & 1.31 & RubisCO \\
\hline 2497 & $0.028^{*}$ & $-2.14^{*}$ & 0.24 & -1.36 & 0.035 & -1.85 & RubisCO \\
\hline $2526^{\#}$ & $0.027^{*}$ & $-2.35^{*}$ & 0.33 & -1.41 & $0.0073^{*}$ & $-3.11^{*}$ & RubisCO \\
\hline 2553 & 0.74 & 1.17 & 0.23 & -1.39 & $0.032^{*}$ & $-2.09^{*}$ & No protein hit \\
\hline 2566 & 0.04 & -1.95 & 0.022 & -1.98 & $0.0044^{*}$ & $-2.63^{*}$ & EFTuA \\
\hline 2568 & 0.062 & -1.54 & 0.3 & -1.29 & $0.0074^{*}$ & $-2.49^{*}$ & RubisCO \\
\hline 2569 & $0.0075^{*}$ & $-2.04^{*}$ & 0.046 & -1.46 & 0.0047 & -1.98 & No protein hit \\
\hline $2589^{\#}$ & $0.023^{*}$ & $-2.27^{*}$ & 0.2 & -1.37 & $0.0049^{*}$ & $-2.45^{*}$ & RubisCO \\
\hline 2602 & 0.58 & 1.22 & $0.0078^{*}$ & $2.5^{\wedge}$ & 0.061 & 1.44 & No MS result \\
\hline $2620^{\#}$ & $0.014^{*}$ & $-2.74^{*}$ & 0.022 & -1.95 & $0.0043^{*}$ & $-2.55^{*}$ & RubisCO \\
\hline 2636 & $0.028^{*}$ & $-2.28^{*}$ & 0.74 & -1.09 & 0.32 & -1.34 & PGK \\
\hline 2655 & $0.022^{*}$ & $-2.34^{*}$ & 0.18 & -1.46 & 0.37 & -1.29 & CBP6 \\
\hline 2668 & $0.022^{*}$ & $-2.13^{*}$ & 0.015 & -1.61 & 0.028 & -1.71 & OEEP1 \\
\hline 2673 & 0.48 & 1.63 & $0.028^{*}$ & $2.03^{\wedge}$ & 0.12 & 1.74 & TKL \\
\hline 2680 & 0.14 & -1.75 & 0.93 & 1.02 & $0.035^{*}$ & $-2.25^{*}$ & RubisCO \\
\hline 2695 & 0.15 & -1.36 & 0.98 & 1.06 & $0.021^{*}$ & $-2.36^{*}$ & RubisCO \\
\hline $2696^{\#}$ & $0.035^{*}$ & $-2.3^{*}$ & 0.2 & -1.42 & $0.041^{*}$ & $-2.18^{*}$ & RubisCO \\
\hline 2720 & $0.039^{*}$ & $-3.49^{*}$ & 0.24 & -1.91 & 0.059 & -2.81 & RubisCO \\
\hline 2790 & 0.08 & -1.84 & $0.025^{*}$ & $-2.38^{*}$ & 0.25 & -1.61 & SATasel \\
\hline 2808 & $0.03^{*}$ & $-2.62^{*}$ & 0.32 & -1.35 & 0.23 & -1.67 & No protein hit \\
\hline
\end{tabular}

* indicates for $t$-test $p$ value lower than 0.05 ; for average ratio it indicates Av. ratio $<-2$; $\wedge$ : Av. ratio $>2$; \# indicates the spot numbers common for 2 studied stresses; ": 1 marked spot was common for all three stress treatments. 
of salinity and drought stress. Salt stress showed 15 spots with higher protein abundance and 20 spots with lower protein abundance compared to control plants. Levels of ATP synthase CF1 alpha chain were significantly changed under all three stress conditions. Oxygen-evolving enhancer protein 2 was significantly changed as a result of salinity. Changes in the abundance of RubisCO activase and one of RubisCO fragments were significant in the same spots only for salt stress and drought stress with no irrigation. Jordan and Chollet [18] stated that the principal role of RubisCO activase is to release inhibitory sugar phosphates, such as ribulose-1,5-bisphosphate, from the active sites of RubisCO to allow its activation by $\mathrm{CO}_{2}$ through carbamylation, whereas Rokka et al. [19] reported that RubisCO activase functions as a chaperone during stress. In addition, Parker et al. [20] stated that the reduction of RubisCO activase due to exposure to $\mathrm{NaCl}$ might be the prime reason of declined photosynthetic activity under $\mathrm{NaCl}$ stress. RubisCO and its fragments were identified in three-monthold seedlings as a result of moderate salt and drought stress [11].

The current results indicate that the high concentration of $\mathrm{NaCl}$ has an inhibitory effect on date palm biosynthesis resulting in reduction in plant growth under high salinity. Mechanisms that contribute to date palm salt tolerance were described through miRNA-mediated gene expressions that are important for adaptation to salinity [21], whereas Carnavale Bottino et al. [22] ascribed salt tolerance in sugarcane to a number of miRNAs involved in salt stress responses in sugarcane.

Drought stress with PEG showed three spots with higher protein abundance and six spots with lower protein abundance compared to control plants. Golldack et al. [23] ascribed changes of the phosphorylation status of cellular proteins to abiotic stress such as drought or salinity. Reactive oxygen species function as an important regulator for many biological processes, such as stress responses, hormone signaling, cell growth, and development [24].

Drought stress with no irrigation did not show any spots with higher protein abundance and showed 19 spots with lower protein abundance compared to control plants. Six spots showed lower protein abundance in salt stress and drought stress due to no irrigation. Only one spot showed lower protein abundance in all three studied stresses. Drought stress primarily results in a reduced rate of photosynthesis $[6,15,16]$. Plant leaf proteome analysis supported this by the lower protein abundance of most photosynthesis enzymes such as ribulose-1,5-bisphosphate carboxylase/oxygenase, oxygen-evolving enhancer protein 2, chloroplastic-like, and cytochrome P450, putative [11]. These findings are supporting those of Loreto et al. [25] who stated that the activity of the photosynthetic electron transport chain is finely tuned to the availability of $\mathrm{CO}_{2}$, and photosystem II activities often decline in parallel by drought stress. In addition, Bota et al. [26] reported that the very severe drought conditions lead to stomatal closures that limit photosynthesis due to a decline in RubisCO activity.

\section{Conclusion}

The proteome analysis of date palm under salinity and drought conditions indicated that both salinity and drought tolerance genes were differentially expressed resulting in high or low protein abundance of the chosen protein spots as a result of exposure to drought and salinity stress condition. In addition, drought stress due to no irrigation caused downexpression of all genes controlling drought tolerance.

\section{Competing Interests}

The authors declare that there is no conflict of interests regarding the publication of this paper.

\section{Acknowledgments}

This project was funded by the National Plan for Science, Technology and Innovation (MAARIFAH), King Abdulaziz City for Science and Technology, the Kingdom of Saudi Arabia, Award no. 11-BIO1545-03. The authors also acknowledge with thanks Science and Technology Unit, King Abdulaziz University, for technical support.

\section{References}

[1] R. Munns and M. Tester, "Mechanisms of salinity tolerance," Annual Review of Plant Biology, vol. 59, pp. 651-681, 2008.

[2] A. Mostek, A. Börner, A. Badowiec, and S. Weidner, "Alterations in root proteome of salt-sensitive and tolerant barley lines under salt stress conditions," Journal of Plant Physiology, vol. 174, pp. 166-176, 2015.

[3] A. Fercha, A. L. Capriotti, G. Caruso et al., "Comparative analysis of metabolic proteome variation in ascorbate-primed and unprimed wheat seeds during germination under salt stress," Journal of Proteomics, vol. 108, pp. 238-257, 2014.

[4] M. W. Yaish and P. P. Kumar, "Salt tolerance research in date palm tree (Phoenix dactylifera L.), past, present, and future perspectives," Frontiers in Plant Science, vol. 6, article 348, 2015.

[5] A. Sambridge, "Drought resistant date palms plan backed by KAUST 2010," Arabian Businessman.com, http://www.arabianbusiness.com/-drought-resistant-date-palms-plan-backed-bykaust-308747.html.

[6] S. Komatsu and Z. Hossain, "Organ-specific proteome analysis for identification of abiotic stress response mechanism in crop," Frontiers in Plant Science, vol. 4, article 71, 2013.

[7] K. G. Rasool, M. A. Khan, A. S. Aldawood, M. Tufail, M. Mukhtar, and M. Takeda, "Identification of proteins modulated in the date palm stem infested with red palm weevil (Rhynchophorus ferrugineus oliv.) using two dimensional differential gel electrophoresis and mass spectrometry," International Journal of Molecular Sciences, vol. 16, no. 8, pp. 19326-19346, 2015.

[8] K. Dadakova, M. Havelkova, B. Kurkova et al., "Proteome and transcript analysis of Vitis vinifera cell cultures subjected to Botrytis cinerea infection," Journal of Proteomics, vol. 119, pp. 143-153, 2015.

[9] M. Mishra, S. Saurabh, R. Maurya, A. Mudawal, D. Parmar, and P. K. Singh, "Proteome analysis of Bemisia tabaci suggests specific targets for RNAi mediated control," Journal of Proteomics, vol. 132, pp. 93-102, 2016. 
[10] A. Ghaffari, J. Gharechahi, B. Nakhoda, and G. H. Salekdeh, "Physiology and proteome responses of two contrasting rice mutants and their wild type parent under salt stress conditions at the vegetative stage," Journal of Plant Physiology, vol. 171, no. 1, pp. 31-44, 2014.

[11] H. A. El Rabey, A. L. Al-Malki, K. O. Abulnaja, and W. Rohde, "Proteome Analysis for Understanding Abiotic Stress (Salinity and Drought) Tolerance in Date Palm (Phoenix dactylifera L.)," International Journal of Genomics, vol. 2015, Article ID 407165, 11 pages, 2015.

[12] Y.-W. Bian, D.-W. Lv, Z.-W. Cheng, A.-Q. Gu, H. Cao, and Y.-M. Yan, "Integrative proteome analysis of Brachypodium distachyon roots and leaves reveals a synergetic responsive network under $\mathrm{H}_{2} \mathrm{O}_{2}$ stress," Journal of Proteomics, vol. 128, pp. 388-402, 2015.

[13] F. Fatehi, A. Hosseinzadeh, H. Alizadeh, T. Brimavandi, and P. C. Struik, "The proteome response of salt-resistant and salt-sensitive barley genotypes to long-term salinity stress," Molecular Biology Reports, vol. 39, no. 5, pp. 6387-6397, 2012.

[14] S. Paul, D. Gayen, S. K. Datta, and K. Datta, "Dissecting root proteome of transgenic rice cultivars unravels metabolic alterations and accumulation of novel stress responsive proteins under drought stress," Plant Science, vol. 234, pp. 133-143, 2015.

[15] P. P. Mohammadi, A. Moieni, S. Hiraga, and S. Komatsu, "Organ-specific proteomic analysis of drought-stressed soybean seedlings," Journal of Proteomics, vol. 75, no. 6, pp. 19061923, 2012.

[16] P. P. Mohammadi, A. Moieni, and S. Komatsu, "Comparative proteome analysis of drought-sensitive and drought-tolerant rapeseed roots and their hybrid F1 line under drought stress," Amino Acids, vol. 43, no. 5, pp. 2137-2152, 2012.

[17] M. W. Yaish, "Proline accumulation is a general response to abiotic stress in the date palm tree (Phoenix dactylifera L.)," Genetics and Molecular Research, vol. 14, no. 3, pp. 9943-9950, 2015.

[18] D. B. Jordan and R. Chollet, "Inhibition of ribulose bisphosphate carboxylase by substrate ribulose 1, 5-bisphosphate," The Journal of Biological Chemistry, vol. 258, no. 22, pp. 13752-13758, 1983.

[19] A. Rokka, L. Zhang, and E.-M. Aro, "RuBisco activase: an enzyme with a temperature-dependent dual function?" Plant Journal, vol. 25, no. 4, pp. 463-471, 2001.

[20] R. Parker, T. J. Flowers, A. L. Moore, and N. V. J. Harpham, "An accurate and reproducible method for proteome profiling of the effects of salt stress in the rice leaf lamina," Journal of Experimental Botany, vol. 57, no. 5, pp. 1109-1118, 2006.

[21] M. W. Yaish, R. Sunkar, Y. Zheng, B. Ji, R. Al-Yahyai, and S. A. Farooq, "A genome-wide identification of the miRNAome in response to salinity stress in date palm (Phoenix dactylifera L.)," Frontiers in Plant Science, vol. 6, article 946, 2015.

[22] M. Carnavale Bottino, S. Rosario, C. Grativol et al., "Highthroughput sequencing of small RNA transcriptome reveals salt stress regulated microRNAs in sugarcane," PLOS ONE, vol. 8, no. 3, article e59423, 2013.

[23] D. Golldack, I. Lüking, and O. Yang, "Plant tolerance to drought and salinity: stress regulating transcription factors and their functional significance in the cellular transcriptional network," Plant Cell Reports, vol. 30, no. 8, pp. 1383-1391, 2011.

[24] J. Bailey-Serres and R. Mittler, "The roles of reactive oxygen species in plant cells," Plant Physiology, vol. 141, no. 2, p. 311, 2006.

[25] F. Loreto, D. Tricoli, and G. di Marco, "On the relationship between electron transport rate and photosynthesis in leaves of the C4 plant Sorghum bicolor exposed to water stress, temperature changes and carbon metabolism inhibition," Australian Journal of Plant Physiology, vol. 22, no. 6, pp. 885-892, 1995.

[26] J. Bota, J. Flexas, and H. Medrano, "Is photosynthesis limited by decreased Rubisco activity and RuBP content under progressive water stress?" New Phytologist, vol. 162, no. 3, pp. 671-681, 2004. 

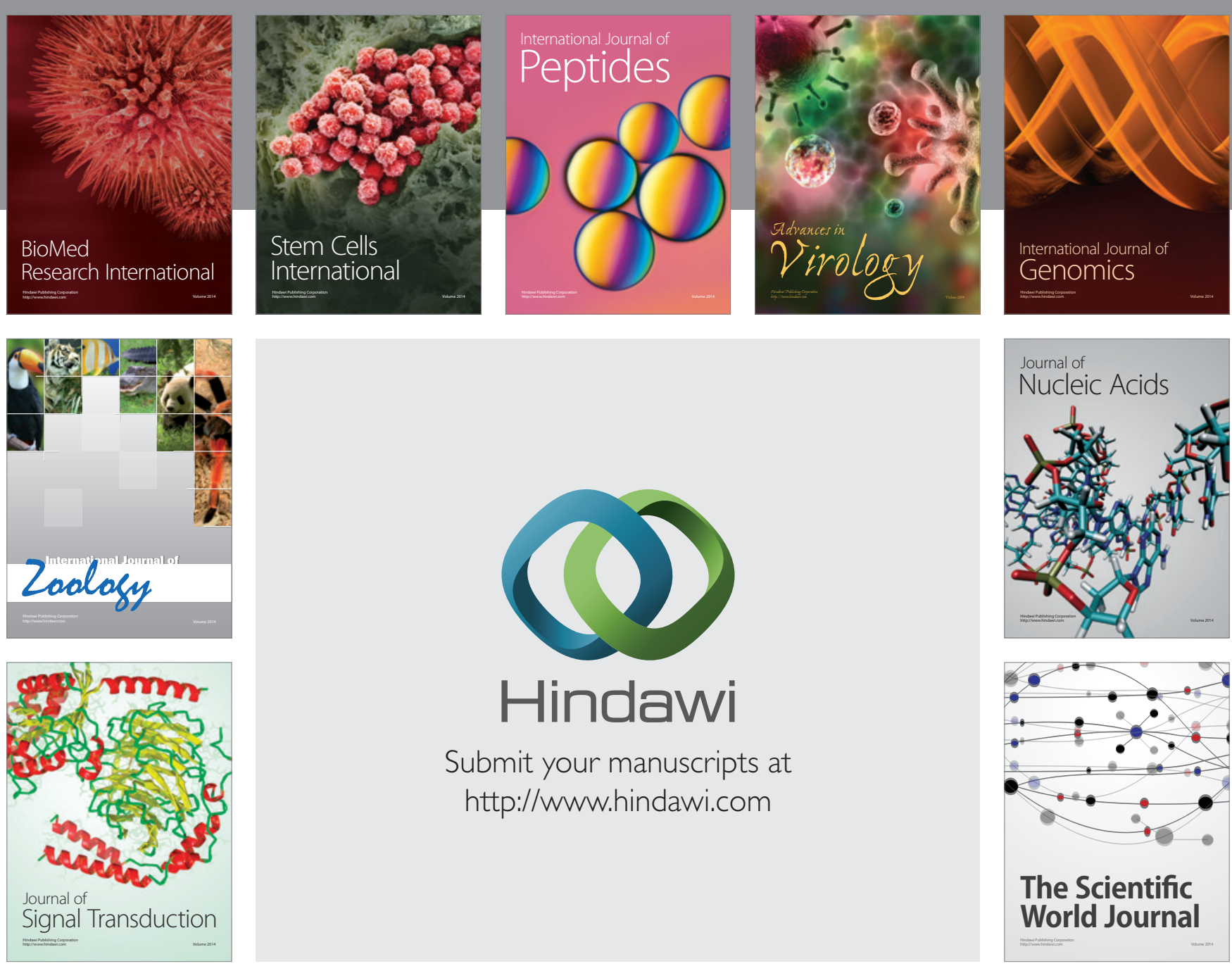

Submit your manuscripts at

http://www.hindawi.com
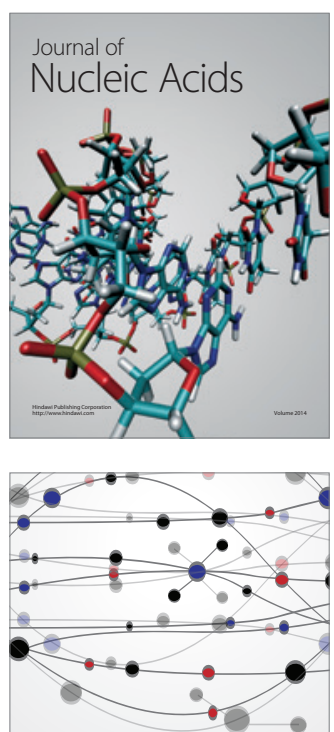

The Scientific World Journal
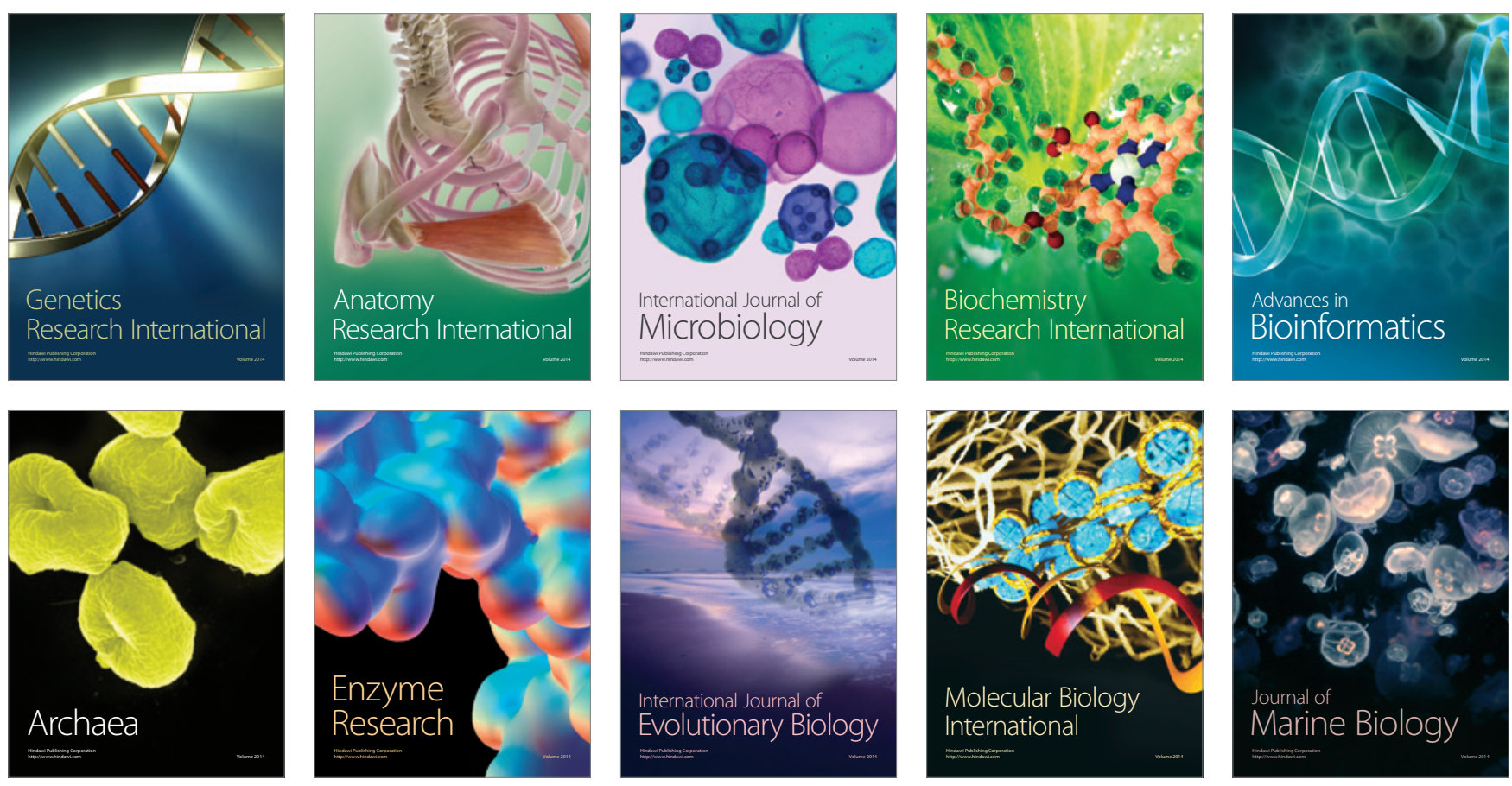